University of New Orleans

ScholarWorks@UNO

10-1989

\title{
Accurate calibration of the four-detector photopolarimeter with imperfect polarizing optical elements
}

\author{
R. M.A. Azzam \\ University of New Orleans, razzam@uno.edu
}

Ali G. Lopez

Follow this and additional works at: https://scholarworks.uno.edu/ee_facpubs

Part of the Electrical and Electronics Commons, and the Optics Commons

\section{Recommended Citation}

R. M. A. Azzam and Ali G. Lopez, "Accurate calibration of the four-detector photopolarimeter with imperfect polarizing optical elements," J. Opt. Soc. Am. A 6, 1513-1521 (1989)

This Article is brought to you for free and open access by the Department of Electrical Engineering at ScholarWorks@UNO. It has been accepted for inclusion in Electrical Engineering Faculty Publications by an authorized administrator of ScholarWorks@UNO. For more information, please contact scholarworks@uno.edu. 


\title{
Accurate calibration of the four-detector photopolarimeter with imperfect polarizing optical elements
}

\author{
R. M. A. Azzam and Ali G. Lopez \\ Department of Electrical Engineering, University of New Orleans, Lakefront, New Orleans, Louisiana 70148
}

Received February 8, 1989; accepted April 11, 1989

\begin{abstract}
The first three columns of the instrument matrix $\mathbf{A}$ of the four-detector photopolarimeter (FDP) are determined by Fourier analysis of the output current vector $\mathrm{I}(P)$ as a function of the azimuth angle $P$ of the incident linearly polarized light. Therefore 12 of the 16 elements of $A$ are measured free of the imperfections of the (absent) quarterwave retarder (QWR). The effect of angular beam deviation by the polarizer is compensated for by taking the average, (1/2) $\left[\mathbf{I}(P)+\mathbf{I}\left(P+180^{\circ}\right)\right]$, of the FDP output at $180^{\circ}$-apart, optically equivalent, angular positions of the polarizer. The remaining fourth column of $\mathbf{A}$ is determined by the FDP's response to the right- and left-handed circular polarization states. Because these states are impossible to generate with an imperfect QWR, a novel procedure is developed. In particular, the response of the FDP to the unattainable right- or left-handed circular polarization state is found by taking the average of the responses of the FDP to an elliptical near-circular state and that state rotated in azimuth by $90^{\circ}$. This calibration scheme is applied to measure A of our prototype FDP of four Si detectors at $\lambda=632.8 \mathrm{~nm}$. A is determined, in external and internal reference frames, free of imperfections in the polarizing optical elements. The FDP, with its uncontaminated A matrix, is used subsequently to evaluate the imperfections of the QWR with the help of an appropriate model.
\end{abstract}

\section{INTRODUCTION}

The four-detector photopolarimeter ${ }^{1-3}$ (FDP) is perhaps the simplest instrument for measuring the state of polarization of a monochromatic or quasi-monochromatic light beam as specified by the four Stokes parameters. ${ }^{4}$ It consists of a stationary arrangement of four photodetectors as that produces an output current vector $\mathbf{I}=\left[\begin{array}{llll}i_{0} & i_{1} & i_{2} & i_{3}\end{array}\right]^{t}$, which is linearly related by

$$
\mathbf{I}=\mathbf{A S}
$$

to the input Stokes vector $\mathbf{S}=\left[S_{0} S_{1} S_{2} S_{3}\right]^{t}$, where the superscript $t$ indicates the transpose. A is a $4 \times 4$ real matrix, called the instrument matrix, which is characteristic of the FDP at a given wavelength.

In this paper we describe a new calibration procedure with which the instrument matrix $\mathbf{A}$ is determined accurately by using an imperfect polarization-state generator (PSG). In particular, we show that $\mathbf{A}$ can be obtained free of the small imperfections of the essential optical elements of the PSG, at least to first order. Furthermore, we show how the FDP (a box of four Si detectors) can subsequently be used with an appropriate model to quantify the PSG imperfections.

\section{DESCRIPTION OF THE SYSTEM}

A computer-controlled FDP has been constructed of four windowless planar-diffused Si photodiodes, operational amplifiers, an analog-to-digital converter, and a personal computer with peripherals, as described elsewhere. ${ }^{2}$

The FDP is calibrated at $\lambda=632.8 \mathrm{~nm}$, using a $1-\mathrm{mW} \mathrm{He}$ Ne laser ${ }^{5}$ whose output beam is nominally circularly polarized externally. The PSG is the polarizing optics of a Gaertner ellipsometer consisting of a Glan-Thompson crystal-prism polarizer (indicated by $\mathrm{P}$ in Fig. 1) and a fixed quartz-plate quarter-wave retarder (QWR) in place of the variable, compound, Babinet-Soleil compensator. ${ }^{2}$ (The nonplanar light path and the gain settings of the operational amplifiers also differ from those used in the first experiment. ${ }^{2}$ ) The state of polarization of light incident upon the FDP is controlled by rotating the polarizer and the QWR around the light beam as an axis. The azimuth angles of these elements, denoted by $P$ and $C$, respectively, are readable on graduated circles to $0.01^{\circ}$ resolution.

\section{CALIBRATION WITH POLARIZATION STATES OF UNEQUAL POWERS}

Because of the inevitable presence of residual linear preferences in the source beam (small but nonzero second and third Stokes parameters) and imperfections in the polarizing optical elements of the PSG, a rotation of the polarizer and the QWR in the beam, to generate different polarization states required for calibration, also changes the power level of the beam. The effect of varying the power level of the beam as the calibration state is changed (or as a result of instability in the source) can be rendered inconsequential by normalization. This is done by dividing the output current vector I of the FDP by the output current $i_{r}$ of a reference detector $D_{r}$ that receives a polarization-independent fraction of the light entering the FDP through a beam splitter (indicated by BS in Fig. 1). There are different possible choices of a beam splitter. In the system that we now use, a pellicle beam splitter is used that also serves as the entrance window of the FDP. The beam splitter is tilted by a small angle $\left(<5^{\circ}\right)$ with respect to the light-beam axis. Because the reference beam is generated by near-normal-incidence reflection from the beam splitter, its power is a fixed fraction of the incident power, independent of the incident polarization. ${ }^{6}$

In calibration, it is the normalized current vector

$$
\mathbf{I}_{n}=\mathbf{I} / i_{r}
$$




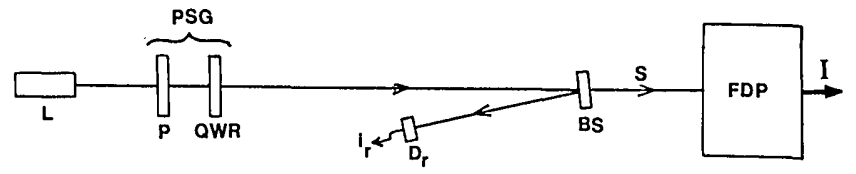

Fig. 1. Schematic diagram of the FDP and the PSG used for its calibration. $L$ indicates the source of a collimated monochromatic unpolarized or circular polarized light beam. $\mathrm{P}$ and $\mathrm{QWR}$ indicate $\mathrm{a}$ linear polarizer and a quarter-wave retarder, respectively. BS indicates a (slightly tilted) beam splitter, and $D_{r}$ indicates a reference detector.

that we use to determine the instrument matrix A. As is noted above, the normalization of Eq. (2) eliminates the effect of a significant source of error. It also justifies the use of a normalized Stokes vector

$$
\mathbf{S}=\mathbf{S}_{n}=\left[\begin{array}{llll}
1 & s_{1} & s_{2} & s_{3}
\end{array}\right]^{t}
$$

to describe the input light to the FDP. The instrument matrix determined by calibration is that which links $\mathbf{I}_{n}$ to $\mathbf{S}_{n}$ :

$$
\mathbf{I}_{n}=\mathbf{A S}_{n}
$$

Because we are interested in polarimetric analysis and not in absolute photometry, determination of $\mathbf{A}$ to within a constant multiplier (or a scale factor) suffices. In what follows, normalization is understood and the subscript $n$ is dropped for simplicity.

\section{RESPONSE OF THE FOUR-DETECTOR PHOTOPOLARIMETER TO INCIDENT LINEARLY POLARIZED LIGHT OF VARIABLE AZIMUTH: DETERMINATION OF THE FIRST THREE COLUMNS OF MATRIX A}

The most significant imperfections of the PSG reside in the QWR. In the visible and near-visible spectrum a crystal polarizer can be used with a very low $\left(\leq 10^{-6}\right)$ extinction ratio, i.e., a nearly ideal polarizer. Therefore it makes sense to determine as much of $\mathbf{A}$ as possible without the QWR. Indeed, the first three columns of $\mathbf{A}$ are determinable with only a linear polarizer in the calibration light beam.

Let $\mathbf{A}$ be written in terms of its columns as

$$
\mathbf{A}=\left[\begin{array}{llll}
\mathbf{A}_{0} & \mathbf{A}_{1} & \mathbf{A}_{2} & \mathbf{A}_{3}
\end{array}\right] \text {. }
$$

The Stokes vector of incident linearly polarized (LP) light of azimuth $P$ is given by

$$
\mathbf{S}_{\mathrm{LP}}(P)=\left[\begin{array}{llll}
1 & \cos 2 P & \sin 2 P & 0
\end{array}\right]^{t} .
$$

If Eqs. (5) and (6) are used in Eq. (1), the response of the FDP to incident LP light (Fig. 2) is obtained in the form

$$
\mathbf{I}_{\mathrm{LP}}(P)=\mathbf{A}_{0}+\mathbf{A}_{1} \cos 2 P+\mathbf{A}_{2} \sin 2 P .
$$

Equation (7) is simple and revealing. It shows that if the output current vector $I(P)$ is recorded as a function of $P$ over one period (a range of $180^{\circ}$ ), as the polarizer is rotated, and is fitted (in a least-squares sense) to the simple Fourier series of Eq. (7) the vectorial Fourier coefficients give directly the first three columns of $\mathbf{A}$. Thus three fourths of the A matrix is determined entirely free of the QWR imperfections because this element is simply absent from the beam.

Figure 3 shows the results that we obtain by applying this procedure to our FDP. The normalized output current of each detector is plotted as a function of the polarizer azi- muth angle $P$ over the range $0 \leq P \leq 180^{\circ}$ in $10^{\circ}$ steps. The diamonds represent the experimental points, and the continuous curves are obtained by a least-squares fit of a scalar function of the form of Eq. (7) to the data. The fit appears nearly perfect for all currents, lending credence to the calibration method and the FDP theory. In fact, the residual rms fitting errors of the four currents are 0.0012, 0.0046, 0.0009 , and 0.0077 , respectively. These numbers are remarkable, considering that the currents are registered with a resolution of $\sim 0.001$, which is limited by our 12-bit analogto-digital converter. The first three columns of $\mathbf{A}$, obtained from the least-squares fitting of the data of Fig. 3, are

$$
\begin{aligned}
& \mathbf{A}_{0}=\left[\begin{array}{llll}
0.746 & 2.433 & 0.694 & 2.597
\end{array}\right]^{t}, \\
& \mathbf{A}_{1}=\left[\begin{array}{llll}
0.157 & -1.180 & -0.244 & -1.934
\end{array}\right]^{t}, \\
& \mathbf{A}_{2}=\left[\begin{array}{llll}
-0.071 & 0.516 & 0.137 & -0.296
\end{array}\right]^{t} .
\end{aligned}
$$

To achieve the excellent results shown in Fig. 3, it was necessary to eliminate the effect of angular beam deviation ${ }^{7}$ introduced by the polarizer (which is $\sim 0.05^{\circ}$ for our GlanThompson prism). This is done by taking the average,

$$
I_{\mathrm{av}}(P)=(1 / 2)\left[\mathrm{I}(P)+\mathrm{I}\left(P+180^{\circ}\right)\right],
$$

of the responses of the FDP recorded at $180^{\circ}$-apart, optically equivalent, positions of the polarizer. The responses of any one detector at these presumably identical positions of the polarizer differ (in a systematic way as the polarizer position is changed) from the average response by as much as $5 \%$. Whereas averaging solves the angular beam deviation problem, it indicates, indirectly, that the field of view of our FDP (in its present form) is restricted. Therefore, light-path stabilization may be required for accurate polarimetric analysis.

\section{RESPONSE OF THE FOUR-DETECTOR PHOTOPOLARIMETER TO INCIDENT RIGHT- AND LEFT-HANDED CIRCULARLY POLARIZED LIGHT: DETERMINATION OF THE FOURTH COLUMN OF MATRIX A}

The remaining (fourth) column of $\mathbf{A}$ is determined from the responses of the FDP to incident right-handed and lefthanded circularly polarized (RCP and LCP, respectively) light. The Stokes vector of such a light is

$$
\mathbf{S}_{\mathrm{CP}}=\left[\begin{array}{llll}
1 & 0 & 0 & \pm 1
\end{array}\right]^{t},
$$

where the + and the - apply for the right- and left-handed circular polarizations, respectively. Substitution of Eqs. (5) and (10) into Eq. (1) gives

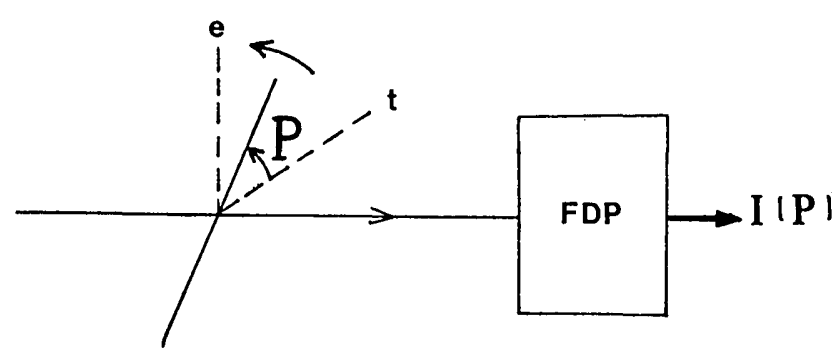

Fig. 2. Calibration of the FDP with LP light of variable azimuth $P$. Fourier analysis of the output current vector $\mathbf{I}(P)$ as a function of $P$ determines the first three columns of the instrument matrix $\mathbf{A}$. 


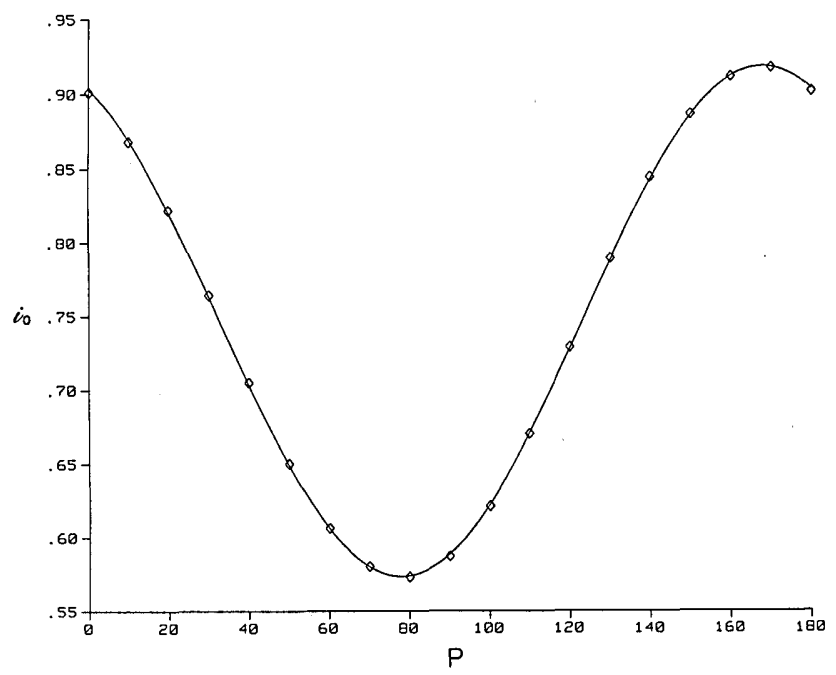

(a)

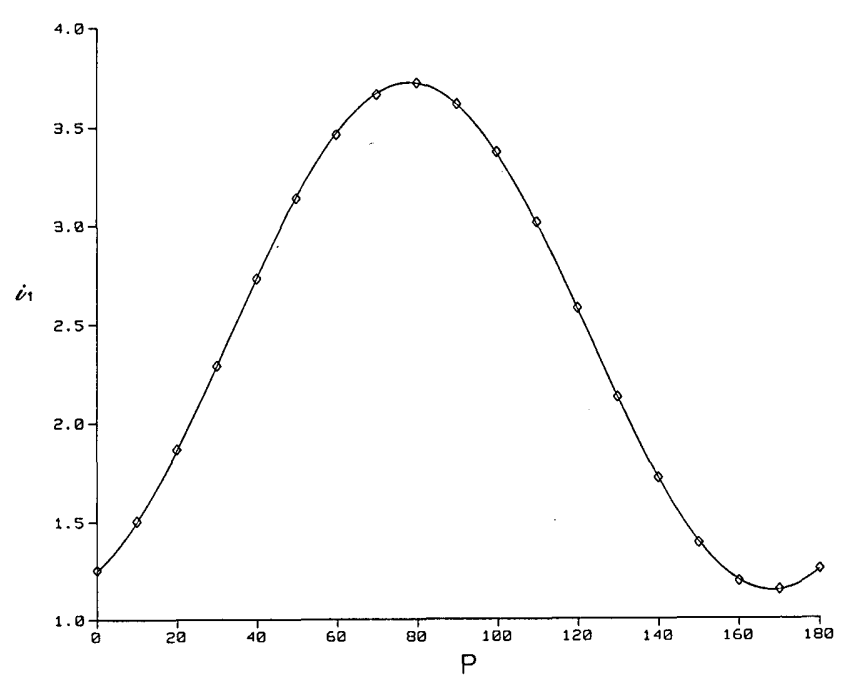

(b)

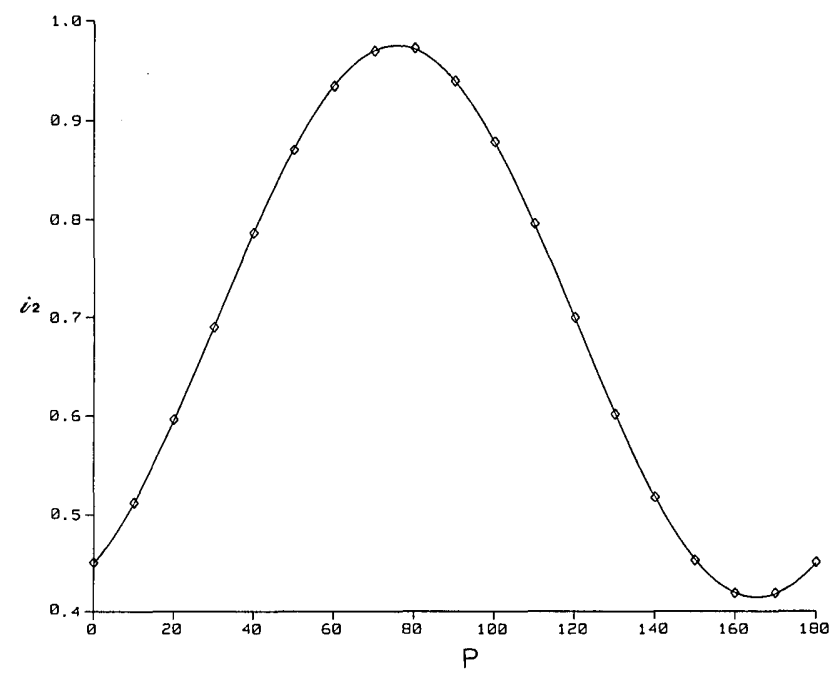

(c)

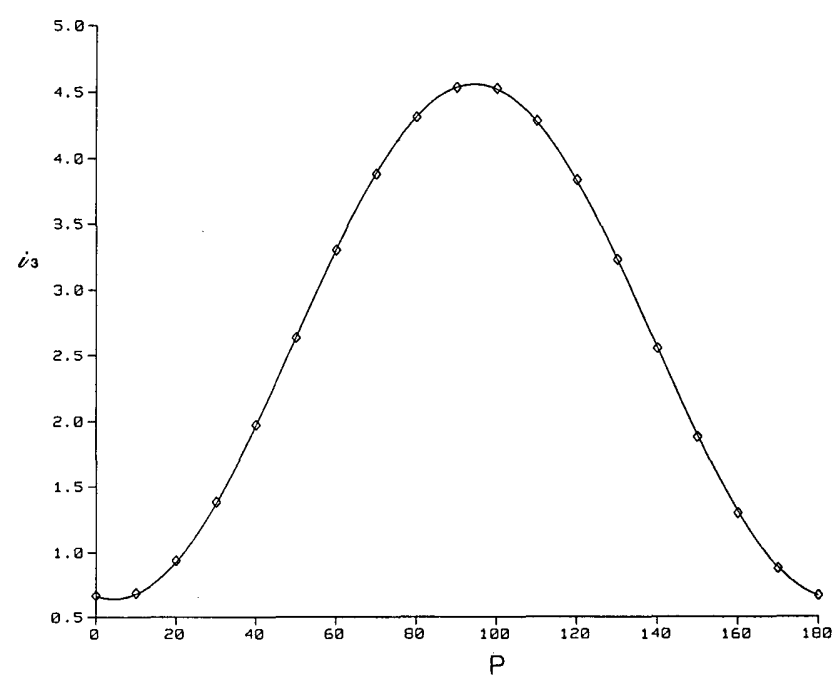

(d)

Fig. 3. The normalized response of each of the four detectors of the FDP as a function of the linear polarizer azimuth $P$ when the calibration scheme of Fig. 2 is used: (a) $i_{0}$, (b) $i_{1}$, (c) $i_{2}$, and (d) $i_{3}$. The diamonds represent the measured response recorded at $10^{\circ}$-apart, equispaced, angular positions of the polarizer over the full range $0 \leq P \leq 180^{\circ}$. The continuous curves are obtained by a least-squares fit of the experimental data points with a function of the form $i_{n}=a_{n 0}+a_{n 1} \cos 2 P+a_{n 2} \sin 2 P$, where $n=0,1,2,3$ and $a_{n 0}, a_{n 1}$, and $a_{n 2}$ specify the first three columns of the instrument matrix $\mathbf{A}$.

$$
\begin{aligned}
& \mathbf{I}_{\mathrm{RCP}}=\mathbf{A}_{0}+\mathbf{A}_{3}, \\
& \mathbf{I}_{\mathrm{LCP}}=\mathbf{A}_{0}-\mathbf{A}_{3},
\end{aligned}
$$

which are the responses of the FDP to RCP light and to LCP light, respectively. From Eqs. (11) the last column of $\mathbf{A}$ is calculated as

$$
\mathbf{A}_{3}=(1 / 2)\left(\mathbf{I}_{\mathrm{RCP}}-\mathbf{I}_{\mathrm{LCP}}\right),
$$

and an independent determination of $\mathbf{A}_{0}$ is obtained:

$$
\mathbf{A}_{0}=(1 / 2)\left(\mathbf{I}_{\mathrm{RCP}}+\mathbf{I}_{\mathrm{LCP}}\right) \text {. }
$$

$\mathbf{A}_{0}$ of Eq. (13) should agree closely with $\mathbf{A}_{0}$ determined from the FDP's response to LP light, as is explained in Section 4. However, Eq. (13) should be used merely as a consistency check and not to revise $\mathbf{A}_{0}$, which is obtained from a plurality of linear states.

The main difficulty in applying the foregoing procedure to determine $\mathbf{A}_{3}$ is that it is practically impossible to generate

the exact RCP and LCP states because of imperfections in the QWR. We have devised a scheme to overcome this problem that is based on the following analysis.

The Stokes vector of a totally polarized light beam with an elliptical vibration of ellipticity angle $\epsilon$ and major-axis azimuth $\theta$ is given by ${ }^{7}$

$$
\mathbf{S}=\left[\begin{array}{llll}
1 & \cos 2 \epsilon \cos 2 \theta & \cos 2 \epsilon \sin 2 \theta & \sin 2 \epsilon
\end{array}\right]^{t} .
$$

We assume that the imperfections of the QWR are small so that the PSG (the polarizer and the QWR) can generate an elliptical near-circular state (ENCS). This ENCS is represented by a point that is near, but not coincident with, the north or south pole of the Poincaré sphere ${ }^{7}$ whose equator represents the LP states. It is convenient to introduce a small parameter

$$
\beta=45^{\circ}-|\epsilon|
$$

to represent the deviation of the ENCS from exact circular- 


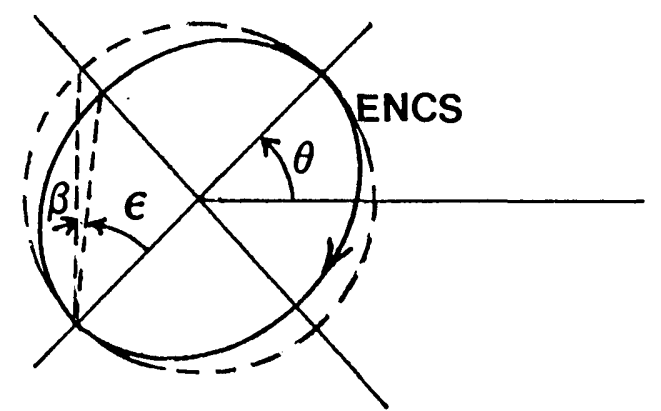

Fig. 4. An ENCS is specified by its major-axis azimuth $\theta$ (unrestricted) and the small deviation $\beta$ of the ellipticity angle $\epsilon$ from $45^{\circ}$ $\left(\beta=45^{\circ}-\epsilon\right)$. The right-handed polarization is indicated by the clockwise arrow, for a beam that travels normal and out of the page toward the reader.

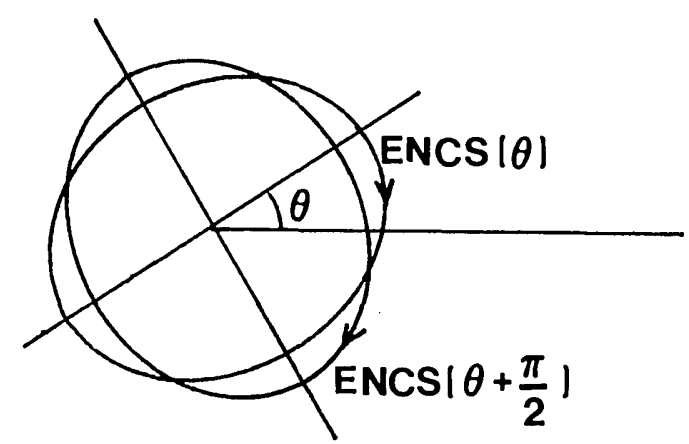

(a)

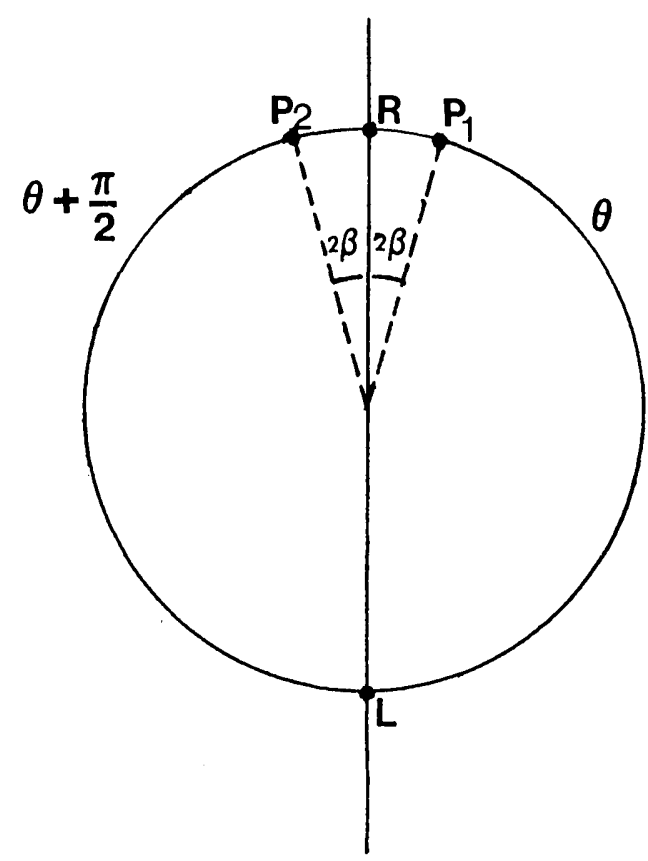

(b)

Fig. 5. (a) An ENCS of azimuth $\theta, \operatorname{ENCS}(\theta)$, and a state produced from it by a $90^{\circ}$ rotation, $\operatorname{ENCS}\left(\theta+90^{\circ}\right)$. (b) Cross section of the Poincare sphere by a plane that contains the polar axis LR and passes through the points that represent the pair of ENCS's in (a). The small angle $\beta$ has the meaning given in Fig. 4. ity (Fig. 4). $\left(\epsilon=+45^{\circ}\right.$ and $-45^{\circ}$ correspond to the RCP and LCP states, respectively.) In terms of $\beta$ and $\theta$, the Stokes vector of the right-handed ENCS is obtained from Eqs. (14) and (15) as

$$
\mathrm{S}_{\mathrm{ENCS}}(\theta)=\left[\begin{array}{llll}
1 & 2 \beta \cos 2 \theta & 2 \beta \sin 2 \theta & 1
\end{array}\right]^{t},
$$

which is valid to first order in the small parameter $\beta$.

Suppose that the polarizer and the QWR are rotated solidly as one unit (preserving the relative orientation between the transmission axis of the polarizer and the fast axis of the QWR at or near $45^{\circ}$ ) by an angle of $90^{\circ}$. This causes the PSG to produce an ENCS of the same $\beta$ but of the orthogonal azimuth $\theta+90^{\circ}$ [Fig. 5(a)]. The Stokes vector of this $90^{\circ}$-rotated ENCS is given by

$$
\mathbf{S}_{\mathrm{ENCS}}\left(\theta+90^{\circ}\right)=\left[\begin{array}{llll}
1 & -2 \beta \cos 2 \theta & -2 \beta \sin 2 \theta & 1
\end{array}\right]^{t}
$$

from Eq. (16).

If the Stokes vectors of Eqs. (16) and (17) are substituted into Eq. (1), we obtain the responses of the FDP to the ENCS and that state rotated in azimuth by $90^{\circ}$. If the average of these two responses is taken, we get

$$
\begin{aligned}
\mathbf{I}_{\mathrm{av}} & =(1 / 2)\left[\mathbf{I}_{\mathrm{ENCS}}(\theta)+\mathbf{I}_{\mathrm{ENCS}}\left(\theta+90^{\circ}\right)\right] \\
& =(1 / 2)\left[\mathbf{A S}_{\mathrm{ENCS}}(\theta)+\mathbf{A S}_{\mathrm{ENCS}}\left(\theta+90^{\circ}\right)\right] \\
& =\mathbf{A}\left\{(1 / 2)\left[\mathbf{S}_{\mathrm{ENCS}}(\theta)+\mathbf{S}_{\mathrm{ENCS}}\left(\theta+90^{\circ}\right)\right]\right\} .
\end{aligned}
$$

From Eqs. (16) and (17) we have

$$
\begin{aligned}
(1 / 2)\left[\mathbf{S}_{\mathrm{ENCS}}(\theta)+\mathbf{S}_{\mathrm{ENCS}}\left(\theta+90^{\circ}\right)\right] & =\left[\begin{array}{llll}
1 & 0 & 0 & 1
\end{array}\right]^{t} \\
& =\mathbf{S}_{\mathrm{RCP}},
\end{aligned}
$$

which is the Stokes vector of the RCP state. Combining Eqs. (18) and (19) gives

$$
\mathbf{I}_{\mathrm{av}}=\mathbf{A} \mathbf{S}_{\mathrm{RCP}}=\mathbf{I}_{\mathrm{RCP}} \text {. }
$$

Equation (20) leads to the following important conclusion: It is possible to determine the response of the FDP to the unattainable exact (right- or left-handed) circular state by finding the average of the responses of the FDP to an ENCS and that state rotated in azimuth by $90^{\circ}$. Notice that $\theta$ and $\beta$ of the ENCS, and hence the PSG imperfections, need not be known.

For specificity, Eqs. (16)-(20) are written for an ENCS near the RCP state or the north pole of the Poincare sphere [Fig. 5(b)]. Of course, the analysis can be repeated readily for an ENCS near the LCP state or the south pole of the Poincaré sphere.

When the above procedure is applied to our FDP, $\mathbf{I}_{\mathrm{RCP}}$ and $\mathbf{I}_{\mathrm{LCP}}$ are obtained, free of PSG imperfections to first order, ${ }^{8}$ and the last column of $A$ is determined by Eq. (12) as

$$
\mathbf{A}_{3}=\left[\begin{array}{llll}
-0.001 & 0.447 & -0.405 & -0.429
\end{array}\right]^{t} \text {. }
$$

This completes the calibration process. The entire matrix $\mathbf{A}$ is listed below for ease of reference:

$$
\mathbf{A}=\left[\begin{array}{rrrr}
0.746 & 0.157 & -0.071 & -0.001 \\
2.433 & -1.180 & 0.516 & 0.447 \\
0.694 & -0.244 & 0.137 & -0.405 \\
2.597 & -1.934 & -0.296 & -0.429
\end{array}\right]
$$




\section{THE INSTRUMENT MATRIX IN EXTERNAL AND INTERNAL REFERENCE FRAMES}

In the course of calibration, the directions of the transmission $(t)$ and extinction (e) axes of the polarizer, when the polarizer scale reads zero $(P=0)$, specify an external (laboratory) reference Cartesian coordinate system with respect to which the Stokes parameters of the calibration light beam, and hence also the instrument matrix $\mathbf{A}=\mathbf{A}_{\text {ext }}$, are defined. The instrument matrix of Eq. (22) is determined with respect to this te system.

When the FDP is removed from the calibration site, it will have no memory of this te laboratory coordinate system.

A unique instrument matrix $\mathbf{A}_{\text {int }}$ can be obtained from $\mathbf{A}_{\text {ext }}$ by a coordinate rotation between the external coordinate system $t e$ and an internal coordinate system $p_{0} s_{0}$, where $p_{0}$ and $s_{0}$ are directions parallel and perpendicular to the first plane of incidence for light reflection at the first detector of the FDP (Fig. 6). $\mathbf{A}_{\text {int }}$ is defined by

$$
\mathbf{I}=\mathbf{A}_{\text {int }} \mathbf{S}_{p_{0} s_{0}},
$$

where $\mathbf{S}_{p_{0} s_{0}}$ is the Stokes vector in the $p_{0} s_{0}$ system that is related to $\mathbf{S}_{t e}$ by

$$
\begin{aligned}
\mathbf{S}_{p_{0} s_{0}} & =\mathbf{R}\left(\alpha_{0}\right) \mathbf{S}_{t e^{*}} \\
\mathbf{R}\left(\alpha_{0}\right) & =\left[\begin{array}{cccc}
1 & 0 & 0 & 0 \\
0 & \cos 2 \alpha_{0} & \sin 2 \alpha_{0} & 0 \\
0 & -\sin 2 \alpha_{0} & \cos 2 \alpha_{0} & 0 \\
0 & 0 & 0 & 1
\end{array}\right]
\end{aligned}
$$

is the Mueller rotation matrix, ${ }^{7}$ and $\alpha_{0}$ is the angle between the directions $p_{0}$ and $t$. From Eqs. (23) and (24), we have

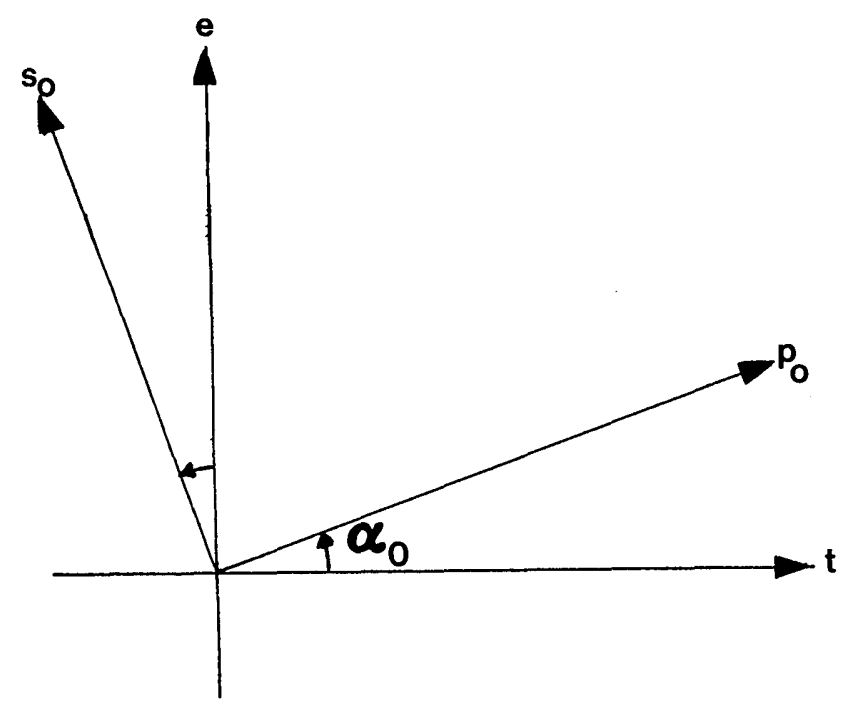

Fig. 6. External and internal reference frames, te and $p_{0} s_{0}$, respectively, with respect to which the Stokes parameters of light and the instrument matrix A of the FDP are defined. $t$ and $e$ represent the directions of the transmission and extinction axes of the polarizer in its reference, $P=0$, orientation. $\quad p_{0}$ and $s_{0}$ are directions parallel and perpendicular to the plane of incidence for light reflection at the first detector inside the FDP. The relative orientation angle $\alpha_{0}$ is determined by a procedure given in the text.

$$
\begin{aligned}
\mathbf{I} & =\left[\mathbf{A}_{\mathrm{int}} \mathbf{R}\left(\alpha_{0}\right)\right] \mathbf{S}_{t e} \\
& =\mathbf{A}_{\mathrm{ext}} \mathbf{S}_{t e},
\end{aligned}
$$

so that

$$
\mathbf{A}_{\text {ext }}=\mathbf{A}_{\text {int }} \mathbf{R}\left(\alpha_{0}\right) .
$$

Postmultiplying both sides of Eq. (27) by $\mathbf{R}\left(-\alpha_{0}\right)$ yields

$$
\mathbf{A}_{\text {int }}=\mathbf{A}_{\text {ext }} \mathbf{R}\left(-\alpha_{0}\right) \text {, }
$$

because $\mathbf{R}\left(\alpha_{0}\right) \cdot \mathbf{R}\left(-\alpha_{0}\right)$ is the $4 \times 4$ identity matrix.

Equations (27) and (28) establish the link between $\mathbf{A}_{\text {int }}$ and $\mathbf{A}_{\text {ext. }}$ From the form of the rotation matrix, Eq. (25), it is evident that $\mathbf{A}_{\text {int }}$ and $\mathbf{A}_{\text {ext }}$ have the same first and last columns; only the middle two columns change in transforming from one reference frame to the other. The angle $\alpha_{0}$ is determined by setting the third element of the first row of $\mathbf{A}_{\text {int }}$ equal to zero, i.e.,

$$
a_{02}{ }^{i}=0,
$$

a condition that follows from the analysis given in Ref. 3 . From Eqs. (25), (28), and (29), one obtains

$$
a_{01}{ }^{e}\left(-\sin 2 \alpha_{0}\right)+a_{02}{ }^{e}\left(\cos 2 \alpha_{0}\right)=0,
$$

where the superscripts $i$ and $e$ refer to the elements of $\mathbf{A}_{\text {int }}$ and $\mathbf{A}_{\text {ext }}$, respectively. The solution of Eq. (30) for $\alpha_{0}$ is

$$
\alpha_{0}=(1 / 2) \arctan \left(a_{02}{ }^{e} / a_{01}{ }^{e}\right) \text {. }
$$

For our FDP at $\lambda=632.8 \mathrm{~nm}, a_{01} e=0.157$ and $a_{02}{ }^{e}=$ -0.071 from Eq. (22); this gives $\alpha_{0}=-12.167^{\circ}$ from Eq. (31). Substitution of the corresponding $\mathbf{R}\left(-\alpha_{0}\right)$ from Eq. (25) and $\mathbf{A}_{\text {ext }}$ from Eq. (22) into Eq. (28) gives

$$
\mathbf{A}_{\text {int }}=\left[\begin{array}{rrrr}
0.746 & 0.172 & 0.000 & -0.001 \\
2.433 & -1.288 & -0.016 & 0.447 \\
0.694 & -0.279 & 0.024 & -0.405 \\
2.597 & -1.640 & -1.067 & -0.429
\end{array}\right],
$$

which is the desired calibration matrix in the internal reference frame $p_{0} s_{0}$.

\section{DETERMINATION OF THE IMPERFECTIONS OF THE QUARTER-WAVE RETARDER}

Having determined the instrument matrix $\mathbf{A}$ of the FDP [Eqs. (22) and (32)] free of the PSG component imperfections, we can work backward to evaluate the more important of these same imperfections, namely, those of the QWR. We can count on the FDP with its reliable $A$ matrix to measure accurately the state of polarization of light that is actually being produced by the PSG. In this curious roundabout way, first the imperfect PSG teaches the FDP, and subsequently the FDP (a box of Si detectors) determines the PSG imperfections!

The (imperfect) QWR is characterized by its slow-to-fast relative amplitude transmittance $T$ and relative phase retardation $\Delta$. The ideal QWR has $T=1$ and $\Delta=90^{\circ}$. We assume the imperfection parameters of the QWR,

$$
\begin{aligned}
& \tau=T-1, \\
& \delta=\Delta-\pi / 2,
\end{aligned}
$$


to be sufficiently small, as befits its role as a calibration component. The QWR is set in a graduated scale mount such that, when the scale reads zero, $C=0$, the fast axis is parallel to (aligned with) the transmission axis of the polarizer in its $P=0$ position. Because this relative alignment is never exact, we introduce a small offset angle $\gamma$,

$$
\gamma=C_{\text {true }}-C
$$

where $C$ and $C_{\text {true }}$ are the scale reading and the true value of the fast-axis azimuth of the QWR measured from the reference $t$ axis.

At the (true) zero azimuth of the fast axis, the Mueller matrix of the QWR is given by 9,10

$$
\mathbf{M}_{\mathrm{QWR}}=\left[\begin{array}{rrrr}
1 & -\tau & 0 & 0 \\
-\tau & 1 & 0 & 0 \\
0 & 0 & -\delta & 1 \\
0 & 0 & 1 & -\delta
\end{array}\right] \text {, }
$$

to first order in $\tau$ and $\delta$ and ignoring an overall amplitude and phase factor in transmission through the QWR.

The Stokes vector at the output of the PSG (consisting of an ideal linear polarizer and the imperfect $Q W R$ ) is given by

$$
\mathbf{S}=\mathbf{R}(-C-\gamma) \mathbf{M}_{\mathrm{QWR}} \mathbf{R}(C+\gamma) \mathbf{S}_{\mathrm{LP}}(P) .
$$

Expansion of Eq. (36) using Eqs. (6), (25), and (35) yields explicit expressions for the Stokes parameters of the light impinging upon the FDP as a function of the azimuth settings $P$ and $C$ and the imperfection parameters $\tau, \delta$, and $\gamma$.

For the purpose of determining $\tau, \delta$, and $\gamma$ it is sufficient to set one optical element at zero azimuth and to rotate the other. Consider first the case of $P=0$ (fixed polarizer) and variable $C$ (rotating QWR). The normalized Stokes parameters obtained from Eq. (36) as functions of $C$ take the forms

$$
\begin{aligned}
& s_{1}=(1 / 2+1 / 2 \cos 4 C)+\Delta s_{1}, \\
& s_{2}=(1 / 2 \sin 4 C)+\Delta s_{2}, \\
& s_{3}=(\sin 2 C)+\Delta s_{3},
\end{aligned}
$$

where the terms within the parentheses on the right-hand sides of Eqs. (37)-(39) give the Stokes parameters transmitted by an ideal PSG and where $\Delta s_{1}, \Delta s_{2}$, and $\Delta s_{3}$ are the Stokes-parameter perturbations caused by the QWR imperfections. The latter can be expressed as Fourier series of $C$ in the following form:

$$
\Delta s_{1}=a_{0}+a_{2} \cos 2 C+a_{4} \cos 4 C+b_{4} \sin 4 C+a_{6} \cos 6 C,
$$

$$
\begin{aligned}
& \Delta s_{2}=d_{2} \sin 2 C+c_{4} \cos 4 C+d_{4} \sin 4 C+d_{6} \sin 6 C, \\
& \Delta s_{3}=e_{2} \cos 2 C+f_{4} \sin 4 C .
\end{aligned}
$$

The 11 Fourier coefficients (or amplitudes) $a_{0}, a_{2}, \ldots, f_{4}$ that appear in Eqs. (40)-(42) are determined in terms of the three imperfections parameters $(\tau, \delta, \gamma)$; hence they are not all independent. The following relations (are supposed to) hold:

$$
\begin{gathered}
a_{0}=-a_{4}=-d_{4}=-\delta / 2, \\
a_{2}=-a_{6}=d_{2} / 3=-d_{6}=-f_{4} / 2=-\tau / 4, \\
b_{4}=-c_{4}=e_{2}=-2 \gamma .
\end{gathered}
$$

Equations (43)-(45) overdetermine the imperfection parameters $\tau, \delta$, and $\gamma$.

The $P=0$, variable- $C$ method is applied by using our FDP. The true normalized Stokes parameters at the output of the PSG are those determined by the well-calibrated FDP by using the inverse relation

$$
\mathbf{S}=\mathbf{A}^{-1} \mathbf{I}
$$

where $I$ is the measured output current vector of the FDP and $\mathbf{A}^{-1}$ is the inverse of the previously determined imperfection-free instrument matrix of Eq. (22):

$$
\mathbf{A}^{-1}=\left[\begin{array}{rrrr}
0.917 & 0.099 & 0.106 & 0.001 \\
1.385 & -0.102 & 0.285 & -0.378 \\
-1.389 & 0.804 & 1.766 & -0.826 \\
0.267 & 0.503 & -1.862 & -0.050
\end{array}\right] .
$$

In Fig. 7 the diamonds represent $s_{1}, s_{2}$, and $s_{3}$ that are obtained from Eq. (46) and the recorded current vector of the FDP, as functions of the fast-axis azimuth reading $C$ of the QWR in $10^{\circ}$ steps of $C$. The continuous curves represent the ideal normalized Stokes parameters of light transmitted by a perfect PSG, which are given by the terms within the parentheses on the right-hand sides of Eqs. (37)-(39).

The (vertical) deviations of the experimental points (FDP-read normalized Stokes parameters) from the ideal curves in Fig. 7 , denoted by $\Delta s_{1}, \Delta s_{2}$, and $\Delta s_{3}$, are plotted separately versus $C$ in Fig. 8. From Fig. 8 it is apparent that $\Delta s_{i}(i=1,2,3)$ represent a predominantly systematic error. This is confirmed by invoking our imperfect-QWR model. By a least-squares analysis, $\Delta s_{i}$ are fitted to Fourier series of the forms of Eqs. (40)-(42), ignoring the theoretical constraints of Eqs. (43)-(45). The results are represented by the continuous curves. The fits for $\Delta s_{1}$ and $\Delta s_{2}$ are excellent, with residual rms errors of 0.0012 and 0.0027 , respectively, which are of the order of the precision limit of our FDP with its 12-bit analog-to-digital converter. Because $\Delta s_{3}$ is nearly an order of magnitude less than $\Delta s_{1}$ and $\Delta s_{2}$, the fit in Fig. 8(c) reproduces only the essential features of the systematic dependence of $\Delta s_{3}$ on $C$. The residual rms error of $\Delta s_{3}$ is 0.0041 .

The Fourier coefficients $a_{2}, a_{4}, \ldots, f_{4}$ that produce the best fit (the continuous curves in Fig. 8) are listed in Table 1. As the reader can readily verify, these coefficients satisfy Eqs. (43) and (44) reasonably well. These equations provide self-consistent and reliable estimates of the intrinsic QWRimperfection parameters $\delta$ and $\tau$ :

$$
\begin{aligned}
\delta & =-0.061( \pm 0.003), \\
\tau & =0.016( \pm 0.002) .
\end{aligned}
$$

Recall that $\delta$ is the deviation of the relative retardance of the QWR from $\pi / 2$ and $\tau$ is the deviation of the slow-to-fast relative amplitude transmittance from 1 .

It should be noted that the above procedure can be used to measure the imperfections of any QWR by using the FDP. (After all, the FDP has no recollection of the specific QWR that was used for its calibration!) This application of the FDP to the characterization of an important and widely used optical element (the QWR) represents a useful byproduct of this study.

Equation (45) is not well satisfied, and the orientational 


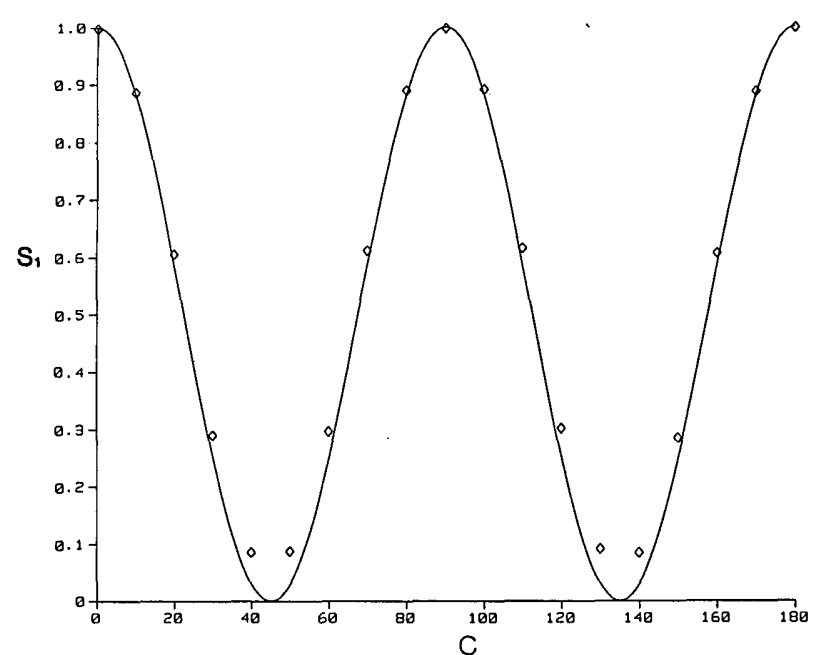

(a)

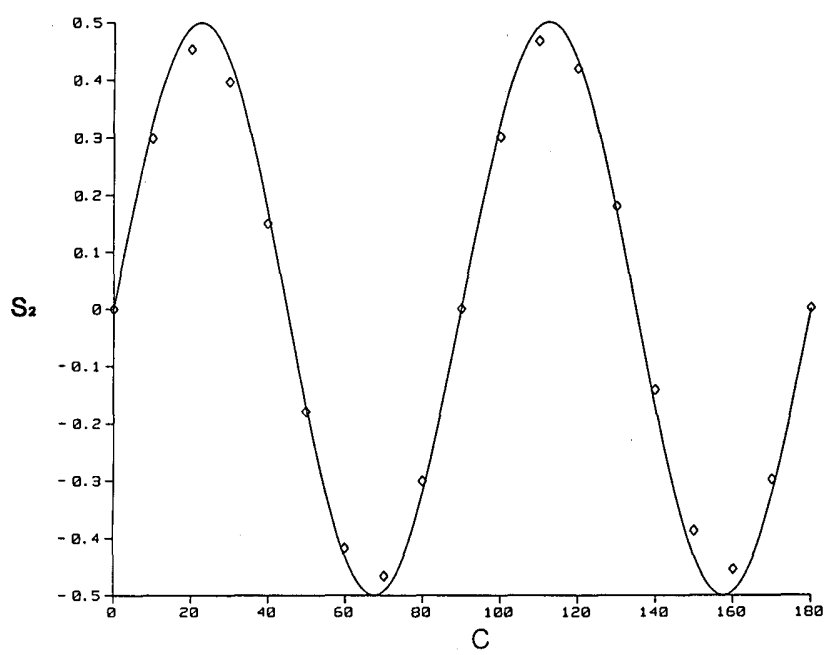

(b)

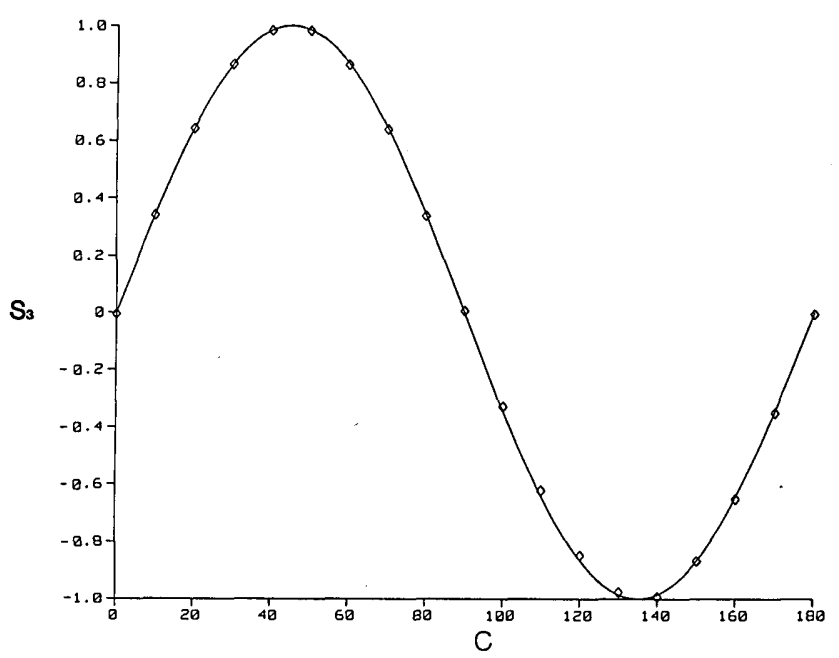

(c)

Fig. 7. Normalized Stokes parameters, (a) $s_{1}$, (b) $s_{2}$, and (c) $s_{3}$, as measured by the well-calibrated FDP (diamonds) and as produced by an ideal PSG (curves). In this test the polarizer is set at zero azimuth $(P=0)$, and the QWR is rotated to vary its fast-axis azimuth $C$ (in $10^{\circ}$ steps). Deviation of the experimental points from the idealized theoretical curves is indicative of imperfections in the QWR.

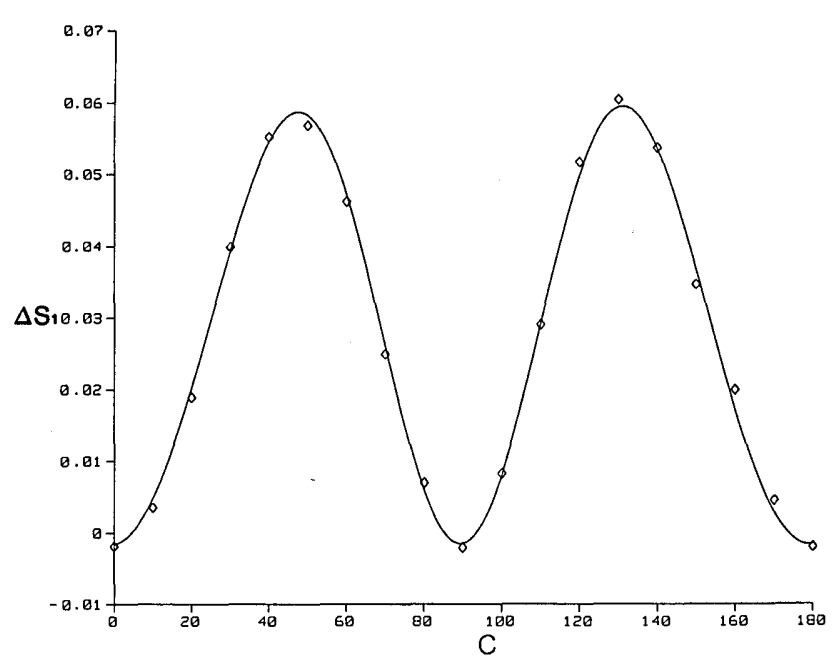

(a)

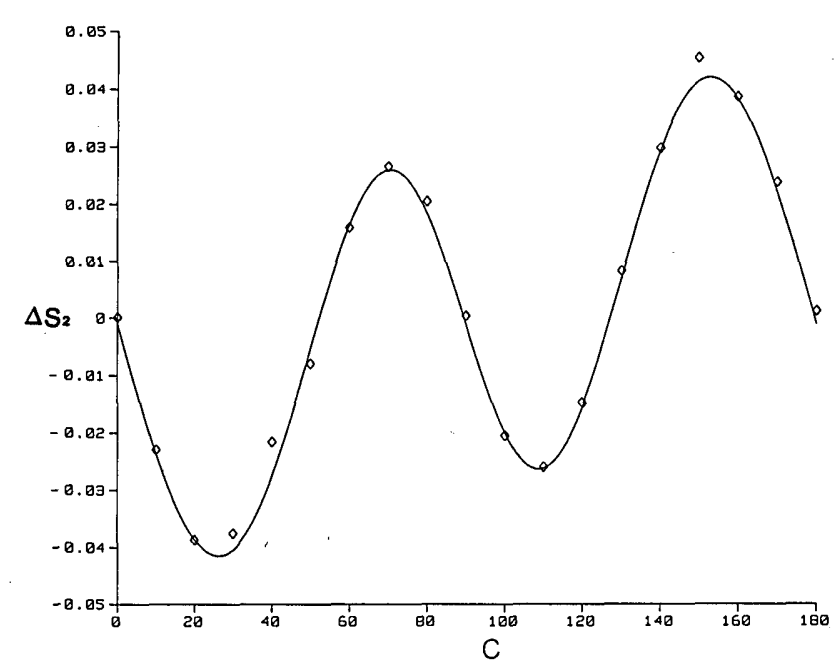

(b)

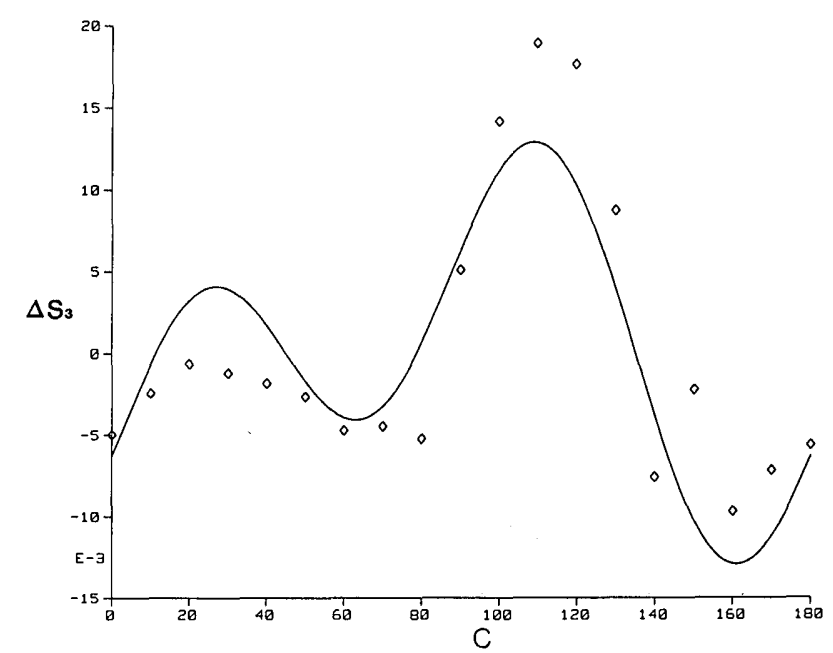

(c)

Fig. 8. Deviations of the FDP-determined normalized Stokes parameters from their ideal values: (a) $\Delta s_{1}$, (b) $\Delta s_{2}$, and (c) $\Delta s_{3}$ are plotted versus the $Q W R$ fast-axis azimuth $C$ with the polarizer set at $P=0$. The discrete points (diamonds) are the measured deviations, and the curves are obtained by a least-squares fit, using Fourier series of the forms given by Eqs. (40)-(42). 
Table 1. Fourier Coefficients for the StokesParameter Perturbations [Eqs. (40)-(42)] in the $P=0$, Variable-C Test $^{a}$

\begin{tabular}{cr}
\hline Coefficient & Value \\
\hline$a_{0}$ & 0.0285 \\
$a_{4}$ & -0.0298 \\
$d_{4}$ & -0.0325 \\
& \\
$a_{2}$ & -0.0035 \\
$a_{6}$ & 0.0035 \\
$d_{2}$ & -0.0144 \\
$d_{6}$ & 0.0036 \\
$f_{4}$ & 0.0081 \\
& \\
$b_{4}$ & 0.0017 \\
$c_{4}$ & -0.0008 \\
$e_{2}$ & -0.0063 \\
\hline
\end{tabular}

a The coefficients are arranged in such a way as to facilitate checking of the theoretical identities of Eqs. (43)-(45) and determination of the imperfection parameters $\tau, \delta$, and $\gamma$ of the QWR. The test is performed on a prototype FDP with four Si detectors at $\lambda=632.8 \mathrm{~nm}$.

misalignment parameter $\gamma$ is ill determined by this particular test.

A more reliable and self-consistent determination of $\gamma$ has been obtained by setting $C=0$ (fixed QWR) and varying $P$ (rotating polarizer). In this case, Eqs. (37)-(45) are replaced by

$$
\begin{aligned}
s_{1} & =(\cos 2 P)+\Delta s_{1}, \\
s_{2} & =(0)+\Delta s_{2}, \\
s_{3} & =(-\sin 2 P)+\Delta s_{3} \\
\Delta s_{1} & =a_{0}^{\prime}+b_{2}^{\prime} \sin 2 P+a_{4}^{\prime} \cos 4 P, \\
\Delta s_{2} & =c_{2}^{\prime} \cos 2 P+d_{2}^{\prime} \sin 2 P \\
\Delta s_{3} & =e_{2}^{\prime} \cos 2 P+f_{4}^{\prime} \sin 4 P
\end{aligned}
$$

and

$$
\begin{aligned}
& a_{0}{ }^{\prime}=-a_{4}{ }^{\prime}=f_{4}{ }^{\prime}=-\tau / 2, \\
& b_{2}{ }^{\prime}=c_{2}{ }^{\prime}=e_{2}{ }^{\prime}=2 \gamma, \\
& d_{2}{ }^{\prime}=-\delta,
\end{aligned}
$$

respectively.

Figure 9 shows $\Delta s_{i}(P)$ versus $P$ for the $C=0$, variable- $P$ method. The coefficients $a_{0}{ }^{\prime}, b_{2}{ }^{\prime}, \ldots, f_{4}{ }^{\prime}$ obtained by leastsquares fitting of Eqs. (53)-(55) to $\Delta s_{i}((i=1,2,3)$ are listed in Table 2. In this case, Eq. (57) is well satisfied, and a good, self-consistent estimate of $\gamma$ is obtained:

$$
\gamma=-0.108^{\circ}\left( \pm 0.013^{\circ}\right) \text {. }
$$

The $C=0$, variable- $P$ method provides a single estimate of the key imperfection parameter $\delta, \delta=-0.057$, in good agreement with the value given in Eq. (48). The three values of $\tau$ that are determined by Eq. (56) are not consistent; however, their average, $\tau=0.011$, agrees reasonably with that of Eq. (49).

To summarize, we recommend that the $P=0$, variable- $C$ test be used for determining the intrinsic imperfections pa-

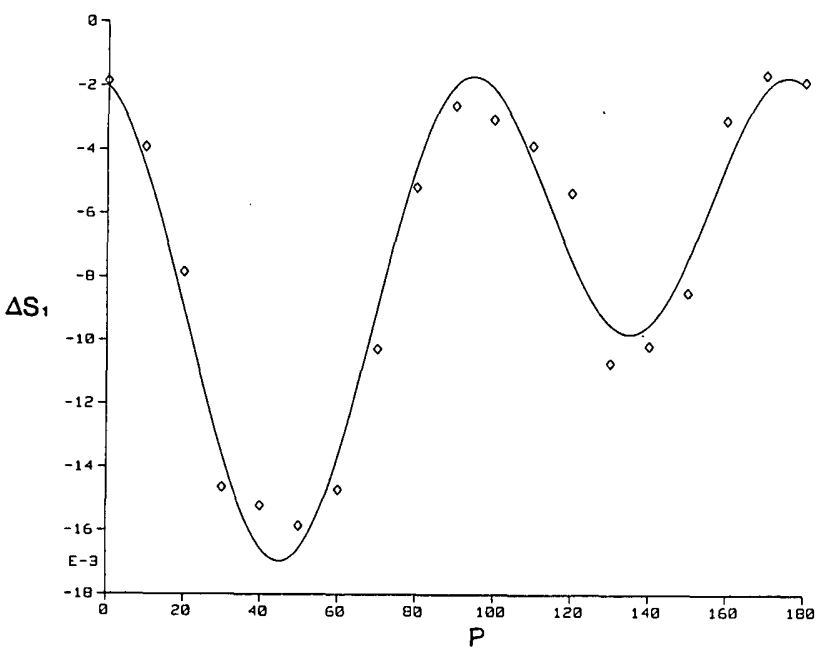

(a)

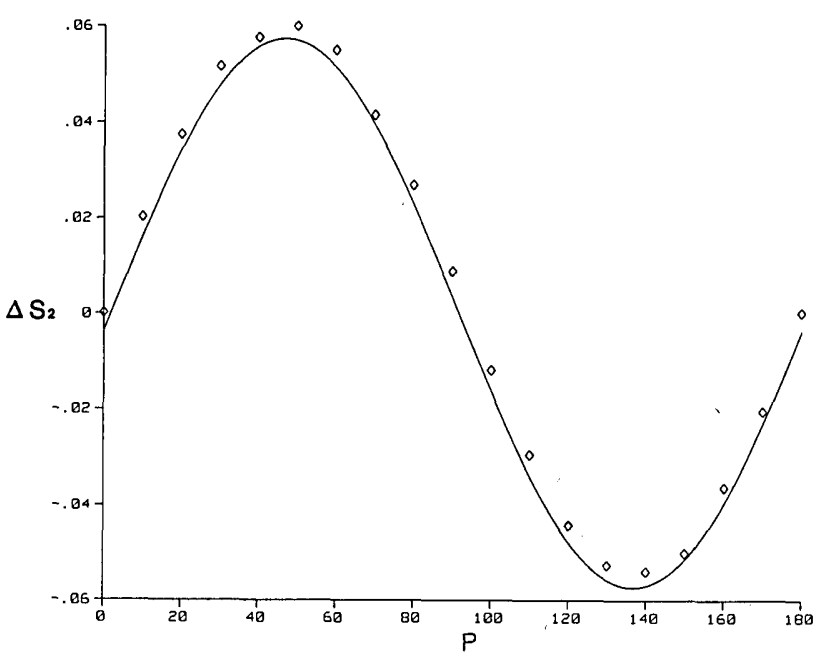

(b)

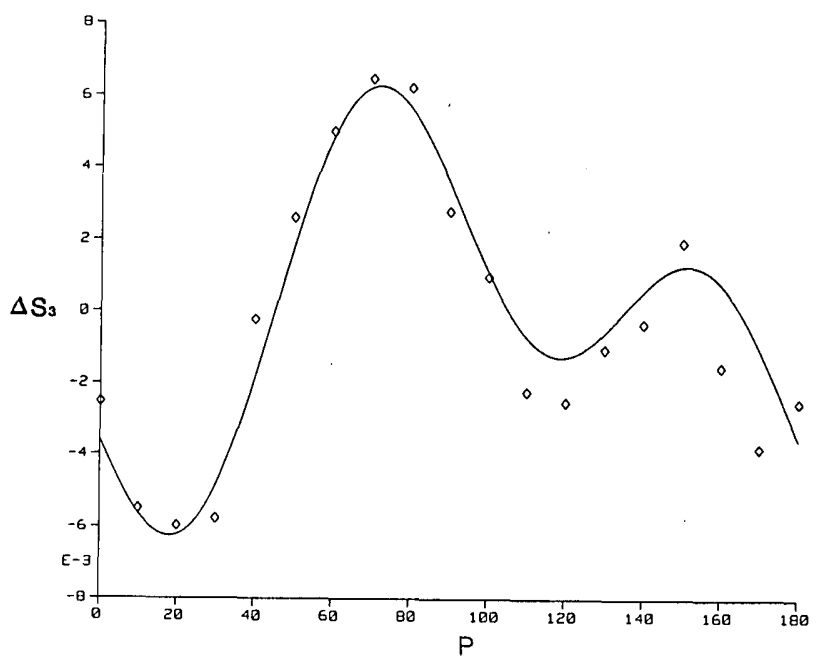

(c)

Fig. 9. Deviations of the FDP-determined normalized Stokes parameters from their ideal values: (a) $\Delta s_{1}$, (b) $\Delta s_{2}$, and (c) $\Delta s_{3}$ are plotted versus the polarizer azimuth $P$ with the QWR set at $C=0$. The Fourier series that fit the experimental data points are given by Eqs. (53)-(55). The residual rms errors in fitting $\Delta s_{1}, \Delta s_{2}$, and $\Delta s_{3}$ are $0.0011,0.0042$, and 0.0016 , respectively. 
rameters $\tau$ and $\delta$ of the QWR. The $C=0$, variable- $P$ test may be performed in addition to obtain a reliable estimate of the fast-axis-azimuth offset (or misalignment) parameter $\gamma$.

\section{APPLICATION TO OTHER FOUR- DETECTOR PHOTOPOLARIMETERS}

The analysis and calibration methods of this paper apply in their entirety to another four-detector polarimeter, also introduced by Azzam. ${ }^{11}$ This is the so-called division-of-amplitude photopolarimeter (DOAP) (see Fig. 10), which uses three beam splitters. One beam splitter (indicated by BS) is conventional, and the other two (indicated by WP) are Wollaston prisms, or equivalent polarizing beam-splitter cubes. The beam splitters break the beam into four beams with each beam intercepted by one detector.

The FDP and the DOAP can be considered from the common electrical engineering black-box point of view. Both are characterized by the same Eq. (1), which links an output current vector $\mathbf{I}$ to an input Stokes vector $\mathbf{S}$.

The only equations that are peculiar to the FDP and that do not apply to the DOAP are Eqs. (29)-(31). Evidently, another criterion needs to be developed for the DOAP to determine the relative orientation of the external calibration reference frame te and the internal (or intrinsic) reference

Table 2. Fourier Coefficients for the StokesParameter Perturbations [Eqs. (53)-(55)] in the $C=0$, Variable-P Test $^{a}$

\begin{tabular}{cr}
\hline Coefficient & \multicolumn{1}{c}{ Value } \\
\hline$a_{0}{ }^{\prime}$ & -0.0076 \\
$a_{4}{ }^{\prime}$ & 0.0057 \\
$f_{4}^{\prime}$ & -0.0035 \\
& \\
$b_{2}{ }^{\prime}$ & -0.0036 \\
$c_{2}{ }^{\prime}$ & -0.0033 \\
$e_{2}{ }^{\prime}$ & -0.0044 \\
$d_{2^{\prime}}$ & 0.0572 \\
\hline
\end{tabular}

\footnotetext{
a The coefficients are arranged in such a way as to facilitate checking of the theoretical identities of Eqs. (56)-(58) and determination of the imperfection parameters $\tau, \delta$, and $\gamma$ of the QWR. The test is performed on a prototype FDP with four Si detectors at $\lambda=632.8 \mathrm{~nm}$.
}

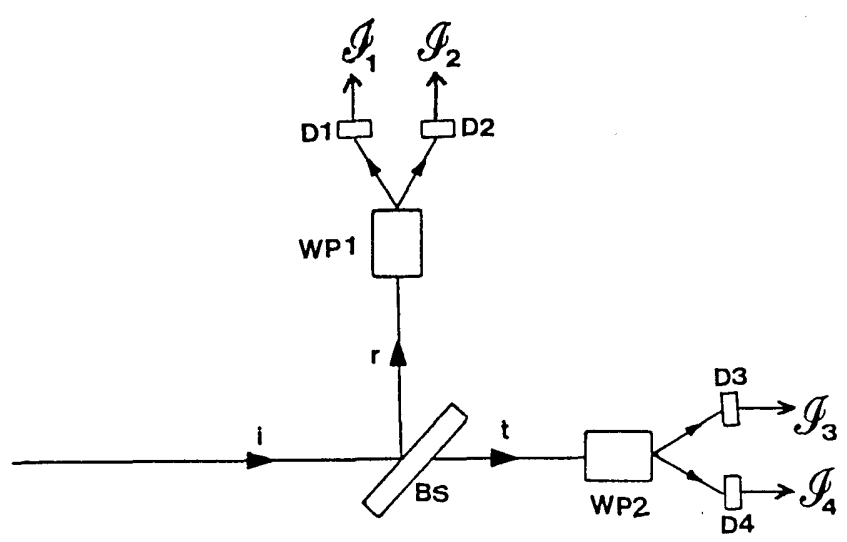

Fig. 10. DOAP, to which much of the analysis of this paper also applies. (See Ref. 11 for details.) coordinate system. The latter consists of the directions parallel $(p)$ and perpendicular $(s)$ to the plane of incidence on the beam splitter indicated by BS, which is the plane of the page in Fig. 10. The search for this criterion, however, falls outside the scope of this paper.

\section{CONCLUSION}

We have presented methods for the accurate calibration of the FDP in the presence of small imperfections in the optical elements of the PSG. We have shown that the effect of these imperfections can be compensated for, or eliminated, so that a clean (uncontaminated) instrument matrix $\mathbf{A}$ is determined. The FDP (a box of four Si detectors) with its good A matrix is then capable of characterizing the imperfections of the same optics used for its calibration.

It has also been noted that much of the analysis and procedures described in this paper also apply to the DOAP. ${ }^{11}$

\section{ACKNOWLEDGMENT}

This study is supported by the National Science Foundation under grant ECS8520035.

\section{REFERENCES AND NOTES}

1. R. M. A. Azzam, "Arrangement of four photodetectors for measuring the state of polarization of light," Opt. Lett. 10, 309-311 (1985); U.S. Patent 4,681,450 (July 21, 1987).

2. R. M. A. Azzam, E. Masetti, I. M. Elminyawi, and F. G. Grosz, "Construction, calibration, and testing of a four-detector photopolarimeter," Rev. Sci. Instrum. 59, 84-88 (1988).

3. R. M. A. Azzam, I. M. Elminyawi, and A. M. El-Saba, "General analysis and optimization of the four-detector photopolarimeter," J. Opt. Soc. Am. A 5, 681-689 (1988).

4. See, for example, M. Born and E. Wolf, Principles of Optics, 5 th ed. (Pergamon, New York, 1975), p. 554.

5. The FDP has also been calibrated and operated at several other wavelengths in the visible spectrum by using a filtered continuum light source. See A. G. Lopez and R. M. A. Azzam, "Fourdetector photopolarimeter (FDP): precision analysis and lowlight-level measurements," in Digest of Optical Society of America Annual Meeting (Optical Society of America, Washington, D.C., 1988), p. 181.

6. R. M. A. Azzam, "Stationary property of normal-incidence reflection from isotropic surfaces," J. Opt. Soc. Am. 72, 1187-1189 (1982).

7. See, for example, R. M. A. Azzam and N. M. Bashara, Ellipsometry and Polarized Light (North-Holland, Amsterdam, 1987).

8. The effect of angular beam derivation of the QWR and by the polarizer is also compensated for by taking the average of four readings of the output current vector $I$ of the FDP corresponding to four optically equivalent settings of the two elements. These settings are obtained by rotating each element by $180^{\circ}$ from a given position, so that $\mathbf{I}=(1 / 4)\left[\mathbf{I}(P, C)+\mathbf{I}\left(P+180^{\circ}, C\right)\right.$ $\left.+\mathbf{I}\left(P, C+180^{\circ}\right)+\mathbf{I}\left(P+180^{\circ}, C+180^{\circ}\right)\right]$. This average is a generalization of that indicated by Eq. (9) for the case in which the polarizer alone is used.

9. R. M. A. Azzam and N. M. Bashara, "Ellipsometry with imperfect components including incoherent effects," J. Opt. Soc. Am. 61, 1380-1391 (1971).

10. P. S. Hauge, "Mueller-matrix ellipsometry with imperfect compensators," J. Opt. Soc. Am. 68, 1519-1528 (1978).

11. R. M. A. Azzam, "Division-of-amplitude photopolarimeter (DOAP) for the simultaneous measurement of all four Stokes parameters of light," Opt. Acta 29, 685-689 (1982); "Beam splitters for the division-of-amplitude photopolarimeter (DOAP)," Opt. Acta 32, 767-777 (1985). 